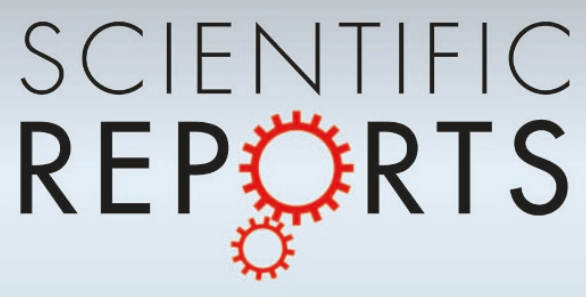

\section{OPEN}

SUBJECT AREAS:

GLYCOBIOLOGY

EMBRYONIC STEM CELLS

STEM-CELL DIFFERENTIATION

GLYCOCONJUGATES

Received

7 June 2013

Accepted

18 December 2013

Published

15 January 2014

Correspondence and requests for materials should be addressed to

H.K. (kitagawa@ kobepharma-u.ac.jp)

\title{
Chondroitin Sulfate Is Indispensable for Pluripotency and Differentiation of Mouse Embryonic Stem Cells
}

\author{
Tomomi Izumikawa, Ban Sato \& Hiroshi Kitagawa
}

Department of Biochemistry, Kobe Pharmaceutical University, Higashinada-ku, Kobe 658-8558, Japan.

Chondroitin sulfate (CS) proteoglycans are present on the surfaces of virtually all cells and in the extracellular matrix and are required for cytokinesis at early developmental stages. Studies have shown that heparan sulfate (HS) is essential for maintaining mouse embryonic stem cells (ESCs) that are primed for differentiation, whereas the function of CS has not yet been elucidated. To clarify the role of CS, we generated glucuronyltransferase-I-knockout ESCs lacking CS. We found that CS was required to maintain the pluripotency of ESCs and promoted initial ESC commitment to differentiation compared with HS. In addition, CS-A and CS-E polysaccharides, but not CS-C polysaccharides, bound to E-cadherin and enhanced ESC differentiation. Multiple-lineage differentiation was inhibited in chondroitinase ABC-digested wild-type ESCs. Collectively, these results suggest that CS is a novel determinant in controlling the functional integrity of ESCs via binding to E-cadherin.

E mbryonic stem cells (ESCs) are pluripotent cells derived from the inner cell mass of the blastocyst-stage embryo $^{1,2}$. In vitro differentiation by formation of free-floating aggregates of ESCs, called embryoid bodies (EBs), is often used for derivation of all three germ layers and can mimic early post-implantation embryonic development ${ }^{3}$. Differentiation and fate commitment of ESCs derived from EBs are controlled by the interaction of the cells with their surrounding microenvironment, of which the extracellular matrix (ECM) is one of the main components. E-cadherin, a major component of the ECM of ESCs, is a cytosolic adaptor that links tight junction transmembrane proteins to the actin cytoskeleton ${ }^{4,5}$. E-cadherin has been implicated in the regulation of signaling pathway hierarchy in $\mathrm{ESCs}^{6,7}$ and in enhanced induced pluripotent stem (iPS) cell generation ${ }^{8-10}$.

Chondroitin sulfate (CS), dermatan sulfate (DS), heparan sulfate (HS), and heparin (Hep) are members of a class of glycosaminoglycans (GAGs) that are found in the ECM on the surface of virtually all cells, including ESCs. Many of the physiological roles of CS/DS-proteoglycans (PGs) and HS-PGs are thought to be attributable to the CS/DS and HS side chains, with core proteins playing the role of scaffolds to make the CS/DS and HS side chains available for binding to a variety of ligands.

$\mathrm{CS} / \mathrm{DS}$ and HS/Hep chains share a common carbohydrate-protein linkage region structure, GlcUA $\beta 1-3 \mathrm{Gal} \beta 1$ 3Gal $\beta 1-4 \mathrm{Xyl} \beta 1$-O-Ser. Glucuronyltransferase I (GlcAT-I) transfers glucuronic acid (GlcUA) from UDP-GlcUA to the trisaccharide-serine, Gal $\beta 1-3 \mathrm{Gal} \beta 1-4 \mathrm{Xyl} \beta 1-\mathrm{O}-\mathrm{Ser}$, and finalizes formation of the common linkage region $^{11,12}$. The repeating disaccharide region [(-4GlcUA $\left.\left.\beta 1-4 \mathrm{GlcNAc} \alpha 1-\right)_{n}\right]$ of HS/Hep is synthesized on the linkage region by the HS co-polymerase complex of EXT1 and EXT2 ${ }^{13,14}$. In contrast, the repeating disaccharide region [(-4GlcUA $\beta 1-3$ GalNAc $\left.\beta 1-)_{n}\right]$ of CS/DS is formed on the linkage region by any two combinations of chondroitin synthase- $1^{15}$, chondroitin synthase- $2^{16}$, chondroitin synthase- $3^{17}$, and chondroitin polymerizing factor $^{18}$. In addition, CS and HS have various structural modifications resulting from sulfation of different positions in the sugar residues ${ }^{19-24}$.

A study with knockout mice showed that CS/DS is required for normal cell division and cytokinesis during early developmental stages ${ }^{25}$, whereas HS is essential for embryonic morphogenesis during later stages of development $^{26,27}$. More recently, using genetic models carrying mutations in genes encoding enzymes for its synthesis, HS, particularly $\mathrm{N}$-sulfation of HS, has been reported to be important for initiation of differentiation and maintenance of the transcriptional and morphological characteristics of $\mathrm{ESCs}^{28-31}$. However, the role of CS in ESCs remains unknown because multiple functionally redundant glycosyltransferases involved in CS/DS synthesis have been cloned $^{32,33}$.

We previously generated GlcAT-I knockout mice ${ }^{25}$. GlcAT-I-deficient mice die by embryonic day 2.5 due to failure of cytokinesis and complete elimination of CS/DS as well as HS. In addition, almost all embryos treated 
with chondroitinase ABC (CSase) die in the two- to eight-cell stage, whereas many heparitinase-treated embryos develop normally to blastocysts. Consequently, we concluded that CS is indispensable for early embryonic cell division in mammals. Notably, from heterozygous intercrossed embryos, $7 \%$ of homozygous GlcAT-I ${ }^{-/-}$ embryos could be identified at the implantation stages ${ }^{25}$. In the current study, we established GlcAT-I-deficient mouse ESCs (mESCs), to determine the role of CS in mESC self-renewal and differentiation. Our results suggest that CS is crucial for the maintenance of ESC pluripotency and differentiation through interactions with Ecadherin.

\section{Results}

GlcAT-I knockout ESCs completely lack CS and HS and fail to initiate differentiation and form EBs. Homozygous $G l c A T-I^{-/-}$ ESCs were established from heterozygous intercrosses. The quantities of CS and HS isolated from GlcAT-I $I^{+/+}, G l c A T-I^{+/-}$, and GlcAT$I^{-1-}$ ESCs were analyzed and measured with high-performance liquid chromatography. CS and HS were completely absent in Glc$A T-I^{-l-}$ ESCs (Table 1 and Supplementary Table S1). Alkaline phosphatase assays performed to measure self-renewal of these cells showed that the level of self-renewal in $G l c A T-I^{-1-}$ ESCs was

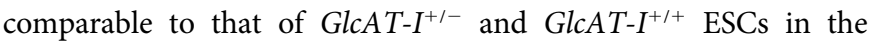
presence of Leukemia inhibitory factor (LIF) (Fig. 1A and B). Realtime RT-PCR and western blot analyses showed increased mRNA and protein expression of two pluripotency genes, Nanog and Sox2,

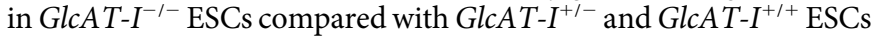
(Fig. 1C and D).

To further explore the role of GAGs in self-renewal, GlcAT-I $I^{+/+}$, GlcAT-I $I^{+-}$, and GlcAT-I $I^{-1-}$ ESCs were cultured in the absence of LIF. A high percentage of $G l c A T-I^{-1-}$ cell colonies remained alkaline phosphatase-positive even in the absence of LIF, showing that Glc$A T-I^{-1-}$ ESCs were unable to exit the self-renewal program (Fig. 2A and B). In addition, GlcAT-I $I^{-1-}$ ESCs retained high expression of Nanog, Oct3/4, and Sox2 (Fig. 2C, D and E).

Next, to verify that GAGs are required for cell fate commitment into multiple lineages, ESCs were differentiated into EBs. GlcAT-I $I^{-\prime-}$ ESCs cultured in static suspension culture exhibited weaker cell-cell interactions than GlcAT-I $I^{+/-}$and $G l c A T-I^{+/+}$EBs and failed to establish characteristic EB morphological features compared to GlcAT$I^{+/-}$and GlcAT-I ${ }^{+/+}$EBs (Fig. 3A). These results appear to be similar to those from EBs that are deficient in HS synthesis $\left(E x t 1^{-1-}\right)^{31}$. During EB differentiation, primitive endodermal (PE) cells develop into the outermost cell layer, similar to the PE cells segregating from the inner cell mass, forming an epithelial layer toward the blastocoel cavity $^{34}$. Upon differentiation of $G l c A T-I^{+/+}$and $G l c A T-I^{+/-}$to EBs,

Table 1 | Disaccharide composition of CS from GlcAT-1+/+, GlcAT-

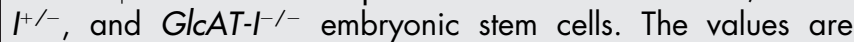
expressed as pmol of disaccharide per $\mathrm{mg}$ of dried homogenate of these cells

\begin{tabular}{|c|c|c|c|}
\hline \multirow[b]{2}{*}{ Disaccharides $^{a}$} & \multicolumn{3}{|c|}{$\mathrm{pmol} / \mathrm{mg}(\mathrm{mol} \%)^{b}$} \\
\hline & GlcAT-I+/+ & GlcAT-I+/- & GlcAT-I/- \\
\hline$\Delta \mathrm{Di}-\mathrm{OS}$ & $14.2 \pm 2.0(21)$ & $5.1 \pm 2.6(14)$ & N.D. \\
\hline$\Delta \mathrm{Di}-6 \mathrm{~S}$ & $6.8 \pm 1.8(10)$ & $2.0 \pm 1.4(5)$ & N.D. \\
\hline$\Delta \mathrm{Di}-4 \mathrm{~S}$ & $46.5 \pm 4.4(68)$ & $29.8 \pm 1.0(80)$ & N.D. \\
\hline$\Delta$ Di-diS $_{D}$ & N.D. ${ }^{c}$ & N.D. & N.D. \\
\hline$\Delta \mathrm{Di}_{\mathrm{i}-\mathrm{diS}}$ & $0.6 \pm 0.3(1)$ & N.D. & N.D. \\
\hline Total (pmol/mg) & $68.1 \pm 8.0$ & $36.9 \pm 5.5$ & N.D. \\
\hline
\end{tabular}

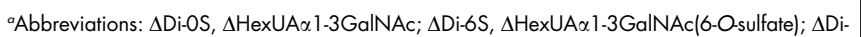

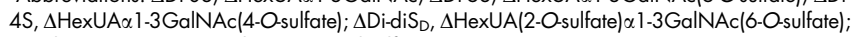

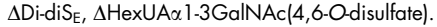

bThe values are the means from three independent experiments.

N.D., not detected. markers of the extraembryonic endoderm (Gata4), early mesoderm (Tbx6, Foxc1, and Brachyury), and ectoderm (Nestin) lineages were upregulated concomitantly with a decline in the transcript and protein levels of pluripotency genes such as Nanog and Oct4 (Fig. 3B, C and D, and also see Supplementary Fig. S1A). In contrast, GlcAT-I-1EBs retained high expression levels of Nanog and Oct4, and lineage markers were undetectable (Fig. 3B, C and D, and also see Supplementary Fig. S1A), indicating that GlcAT-I-1- ESCs failed to differentiate into multiple lineages.

The sulfation pattern of CS regulates EB differentiation. If CS is indispensable for cell fate commitment and cell-cell interactions in EBs, digestion of CS on wild-type (Wt) ES cells may also influence EB formation. In fact, treatment of Wt ESCs with CSase had marked effects on EB formation, although heparitinase-treated ESCs seemed to develop normally to EBs (Fig. 4A, C and D, and Supplementary Fig. S1B), indicating that CS chains on the cell surface were involved in EB differentiation. The complete and selective digestion of GAG chains was confirmed by immunofluorence image using anti-CS (LY111) or anti-HS (Hepss-1) monoclonal antibodies (Fig. 4B). Next, to investigate the importance of specific sulfation sequences on CS chains in ESC differentiation, the CS disaccharide composition was analyzed during the differentiation of ESCs into EBs. Differentiating EBs exhibited an increase in the total content of CS disaccharides over time (Supplementary Table S2). Additionally, although the amount of $\triangle \mathrm{HexA}-\mathrm{GalNAc}(4-\mathrm{O}$-sulfate, 6-O-sulfate) (CS-E-unit) and $\triangle \mathrm{HexA}-\mathrm{GalNAc}(4-\mathrm{O}$-sulfate) (CS-A-unit) markedly increased, that of $\Delta \mathrm{HexA}-\mathrm{GalNAc}$ and $\Delta \mathrm{HexA}-\mathrm{GalNAc}(6-O-$ sulfate) (CS-C-unit) decreased slightly as differentiation progressed (Supplementary Table S2). These results suggest that CS-A and CS-E chains may be important for EB differentiation. Thus, to confirm the role of CS in EB differentiation, additional analyses of $G l c A T-I^{-1-} \mathrm{ES}$ cells cultured in the presence of CS-A, CS-C, CS-E, or Hep polysaccharides were performed. As shown in Fig. 4E and Supplementary Fig. S2A and C, addition of CS-A or CS-E polysaccharides restored the EB size and proliferative capacity, whereas addition of CS-C or Hep did not. Furthermore, addition of CS-A or CS-E polysaccharides to GlcAT-I ${ }^{-1-}$ EB cultures rescued the differentiation of these cells into PE (Fig. 4E, F and G and Supplementary Fig. S1A). These results indicate that CS-A and CS-E may be essential factors in EB formation.

E-cadherin interacts directly with CS-A and CS-E. We have recently demonstrated that CS-E fine-tunes osteoblast differentiation via extracellular signaling-regulated kinase (ERK)1/2, Smad3, and Smad $1 / 5 / 8$ signaling by binding to $\mathrm{N}$-cadherin and cadherin- $11^{35}$. In fact, CS-E interacts with $\mathrm{N}$-cadherin and cadherin-11 through calcium-dependent adhesion, whereas CS-A does not. In addition, E-cadherin-null ESCs fail to form EBs and remain undifferentiated even in the absence of $\mathrm{LIF}^{7}$. Therefore, we hypothesized that interaction between CS with a specific structure such as CS-A or CS-E and $\mathrm{E}$-cadherin is required for $\mathrm{EB}$ formation and differentiation. Thus, the interaction of various CSs and E-cadherin was examined using the surface plasmon resonance biosensor, BIAcore. As shown in Fig. 5A and Supplementary Table S3, the CS-E polysaccharide bound strongly to E-cadherin only in the presence of calcium. In addition, CS-A also bound to E-cadherin only in the presence of calcium, whereas CS-C polysaccharide did not. Thus, CS-A and CS-E interacted with E-cadherin in a calcium-dependent manner.

We next examined whether CS colocalized with E-cadherin in ESCs. Double immunostaining of Wt ESCs using anti-CS (LY111) or anti-HS (Hepss-1) monoclonal antibodies and anti-E-cadherin was carried out. CS colocalized with E-cadherin on the cell surface and in the intercellular junctions (Fig. 5B), whereas HS was localized diffusely on the cell surface and was partially colocalized with Ecadherin (Fig. 5C). 
A
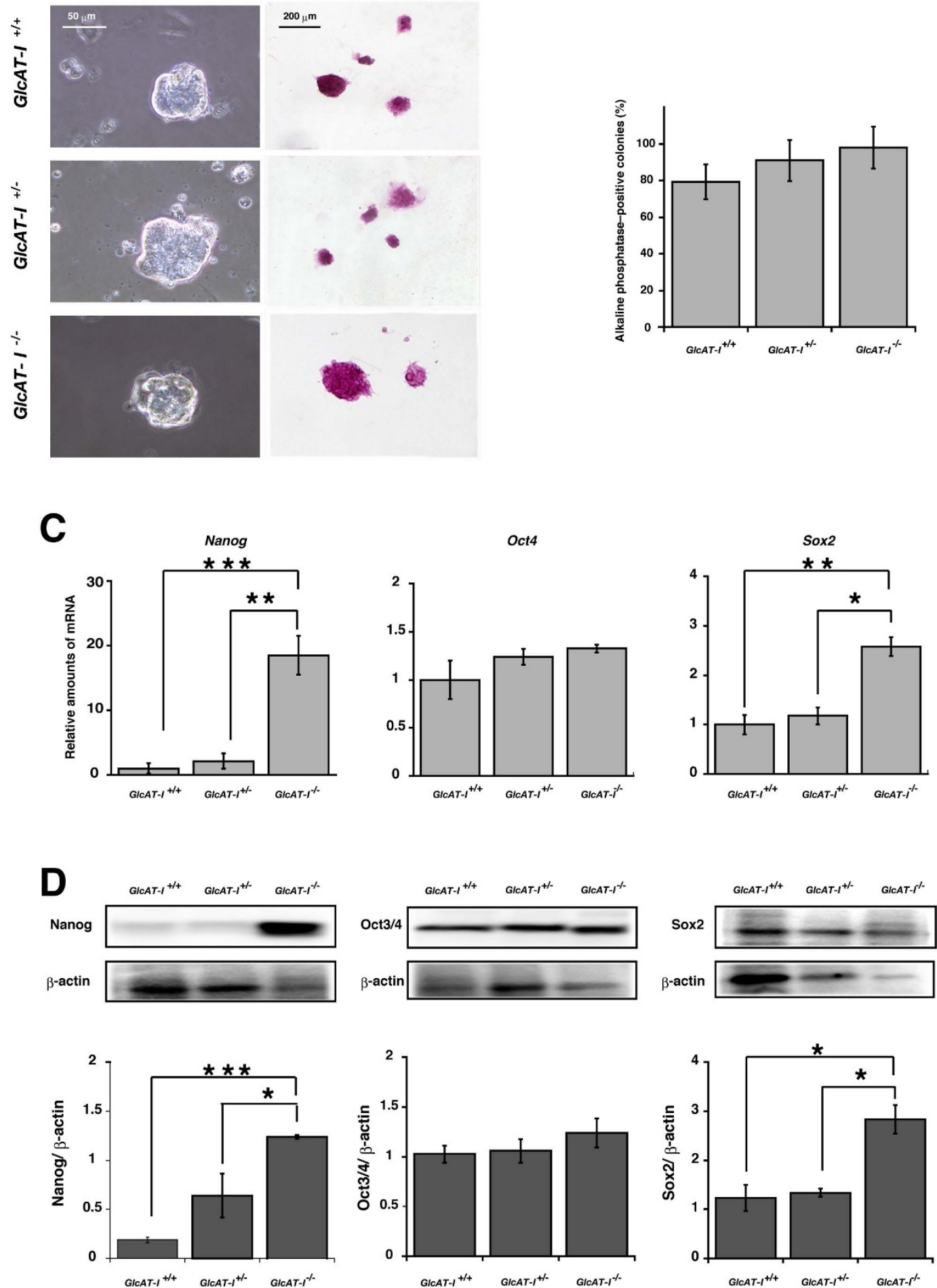

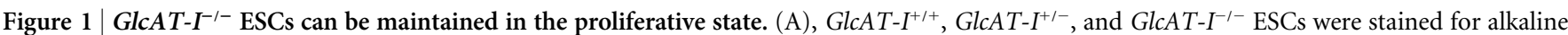
phosphatase activity in the presence of LIF. B, ESCs were cultured for $5 \mathrm{~d}$ and then plated at clonal density. The percentage of alkaline phosphatasepositive colonies was quantitated. Error bars indicate the S.E. for triplicates of the same experiment, which is representative of at least three independent experiments. C, Real-time PCR results for pluripotency genes. Total RNA was extracted from ESCs cultured in feeder-free conditions. ${ }^{*} \mathrm{P}<0.01$,

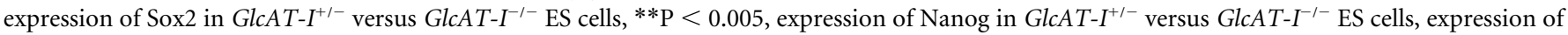

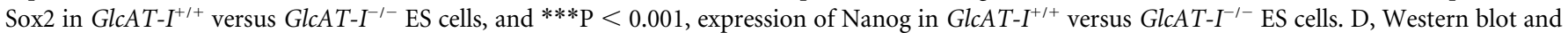
quantitative analysis of pluripotency markers, Nanog, Oct3/4, and Sox 2 in ESCs cultured in the presence of LIF. $\beta$-actin was used as a loading control. The gels have been run under the same experimental condition. The blot image was cropped around the region of interest. The results are representative of at least three independent experiments. Statistical analysis was carried out by Student's $t$ test. $* \mathrm{P}<0.01$ and $* * * \mathrm{P}<0.001$. 
A

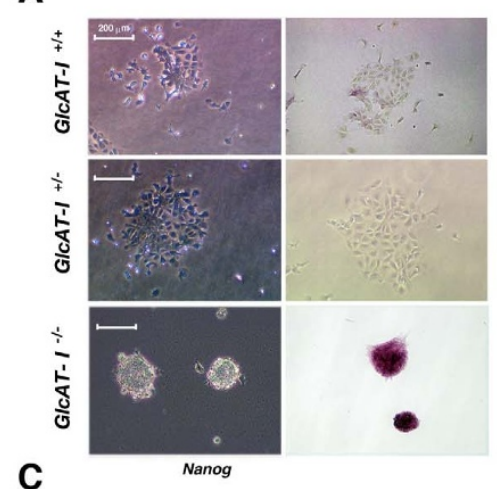

C

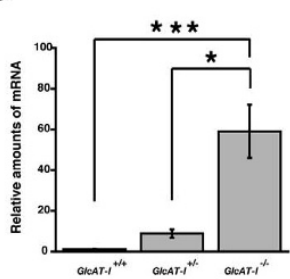

B

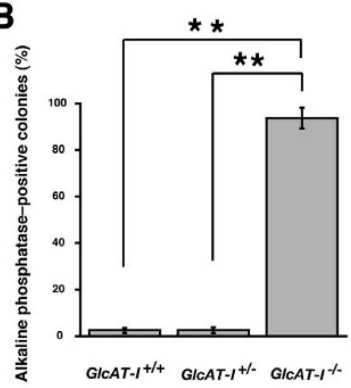

D
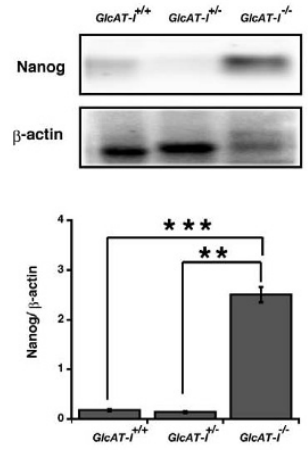
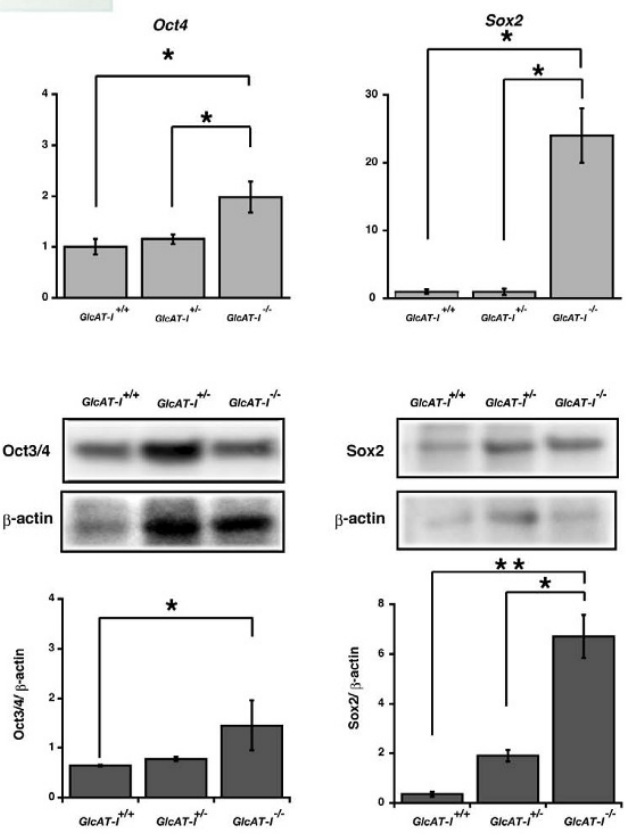

E
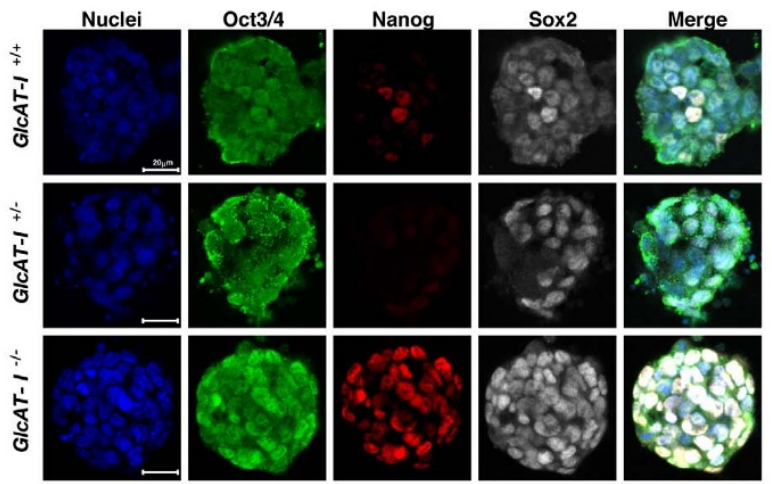

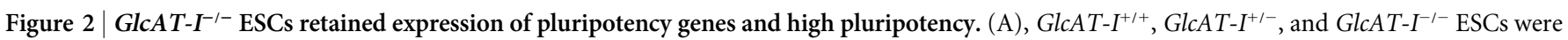
plated at clonal density, cultured in the absence of LIF for $5 \mathrm{~d}$, and stained for alkaline phosphatase. (B), The percentage of undifferentiated colonies was examined with alkaline phosphatase assays. Error bars indicate the S.E. for triplicates of the same experiment, which is representative of at least three

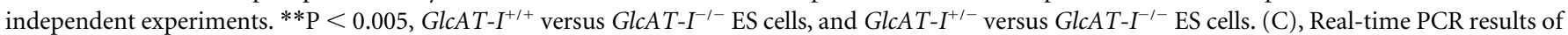
pluripotency marker genes in ESCs cultured in the absence of LIF. GAPDH transcripts were used as an internal control. *P $<0.01$, expression of Nanog in

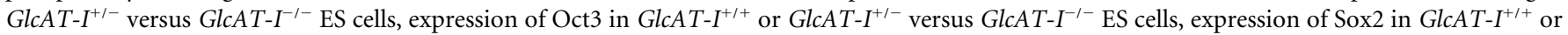

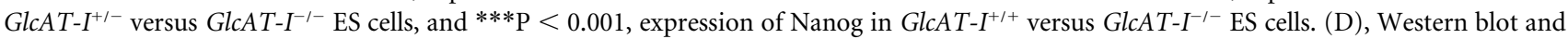
quantitative analysis of pluripotency markers, Nanog, Oct3/4, and Sox 2 in ESCs cultured in the absence of LIF. $\beta$-actin was used as a loading control. The gels have been run under the same experimental condition. The blot image was cropped around the region of interest. The results are representative of at least three independent experiments. Statistical analysis was carried out by Student's $t$ test. ${ }^{*} \mathrm{P}<0.01,{ }^{* *} \mathrm{P}<0.005$ and ${ }^{* * *} \mathrm{P}<0.001$. (E), The expression

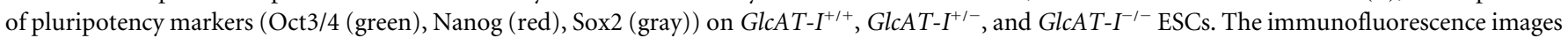

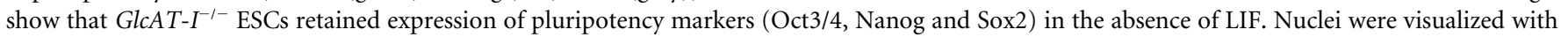
DAPI staining (blue). 
A
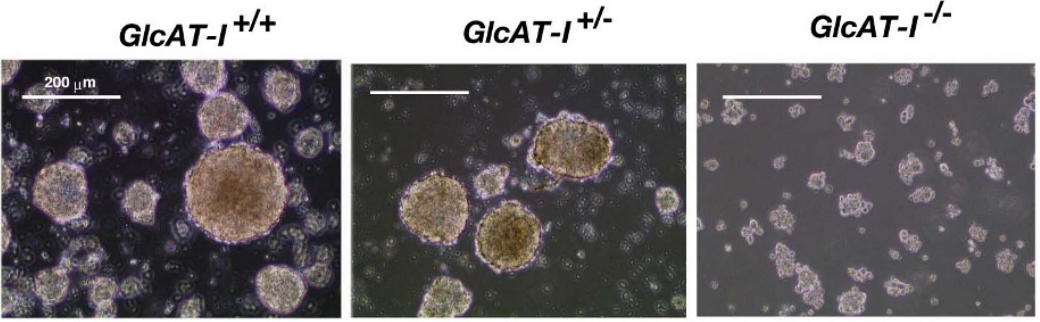

B
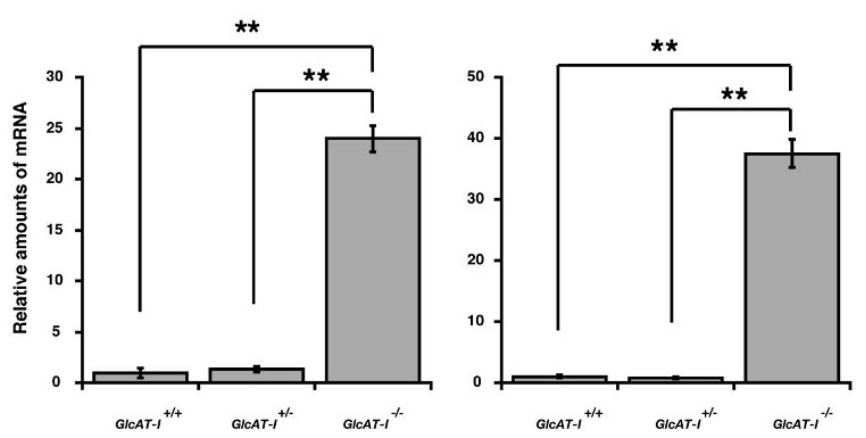

c

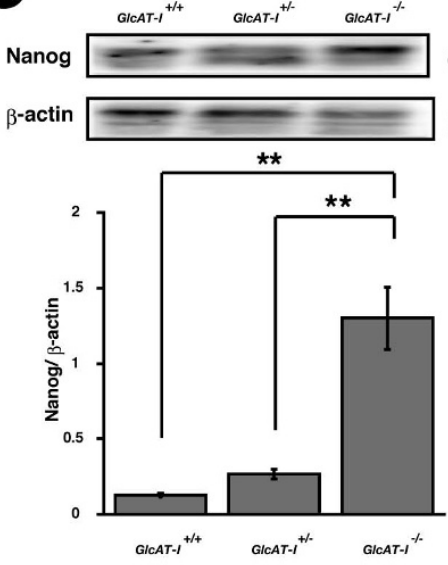

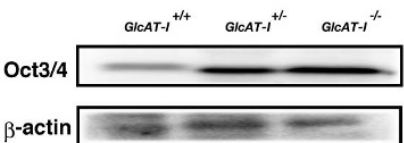

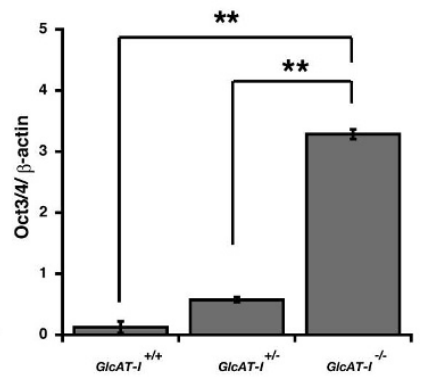

D
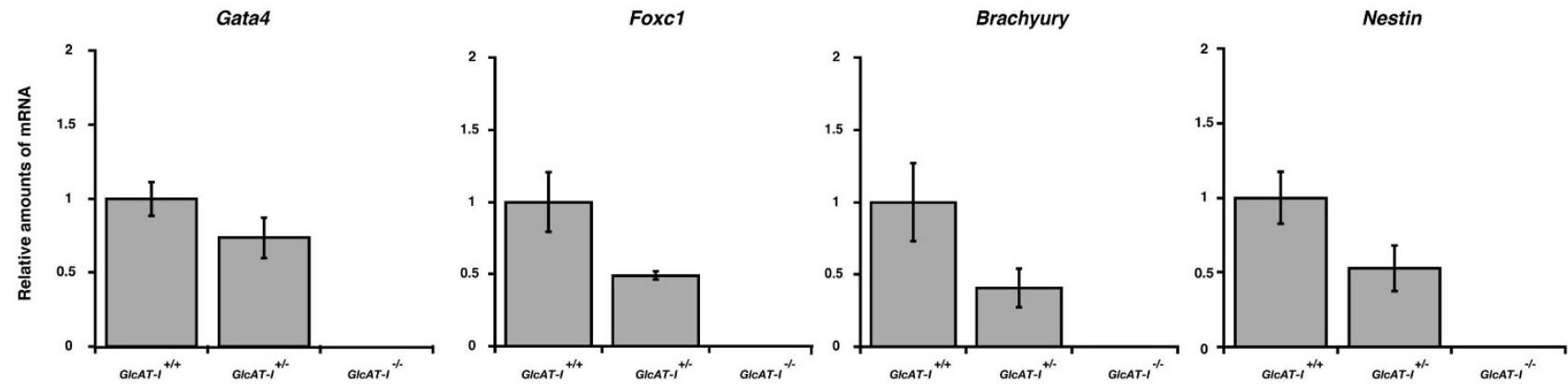

Figure $3 \mid$ GlcAT-I ${ }^{-1-}$ ESCs fail to differentiate into EBs. (A), Phase-contrast microscopy of day 4 EBs. (B), Real-time PCR results for pluripotency marker genes, Nanog and Oct4 during in vitro differentiation of ESCs. GAPDH transcripts were used as an internal control. (C), Western blot and quantitative analysis of pluripotency markers, Nanog and Sox 2 during in vitro differentiation of ESCs. $\beta$-actin was used as a loading control. The gels have been run under the same experimental condition. The blot image was cropped around the region of interest. The results are representative of at least three independent experiments. (D), Real-time PCR results for differentiation marker genes, Gata4, Foxc1, Brachyury, and Nestin during in vitro differentiation of ESCs. GAPDH transcripts were used as an internal control. Statistical analysis was carried out by Student's $t$ test. ${ }^{*} \mathrm{P}<0.01$ and $* * \mathrm{P}<0.005$.

Using a neutralizing antibody against E-cadherin, we further analyzed whether the effect of exogenous CS-A or CS-E on EB formation was dependent on E-cadherin. Addition of CS-A in the presence of anti-E-cadherin antibody (E-cad Ab) did not restore EB size (Fig. 6A and B) or the proliferative capacity (Supplementary Fig. S2C) and did not induce ESC differentiation into the PE layer (Fig. 6C and D). In

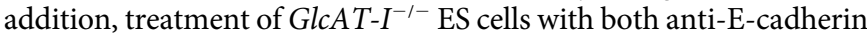
antibody and CS-E failed to establish characteristic EB morphology and cell proliferation (Supplementary Fig. S2B and C). In contrast, treatment of GlcAT-I ${ }^{-l-}$ ES cells with only anti-E-cadherin antibody had no effect on EB size or proliferative capacity compared with untreated GlcAT-I ${ }^{-1-}$ ES cells (Supplementary Fig. S2B and C). These results indicated that CS chains are involved in controlling initial differentiation of ESCs by binding to E-cadherin. Of note, Ecadherin expression was increased in GlcAT-I ${ }^{-1-}$ ES cells compared with $G l c A T-I^{+/+}$ES cells (Supplementary Fig. S3). Consistent with this, the cell attachment rate was about 2 -fold higher in GlcAT-I ${ }^{-1-}$ ES cells compared with wild-type ES cells, indicating that the adhesive function of E-cadherin was not disrupted in $G l c A T-I^{-1-}$ ES cells (Supplementary Fig. S4). Taken together, the data suggest that CS-A and CS-E are selective ligands for a potential CS receptor, E-cadherin, leading to ES cell differentiation. 
A

Wt

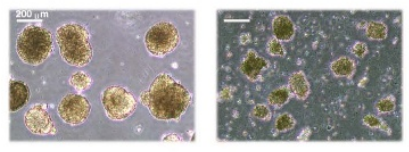

HSase

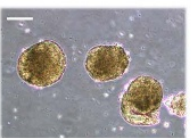

B

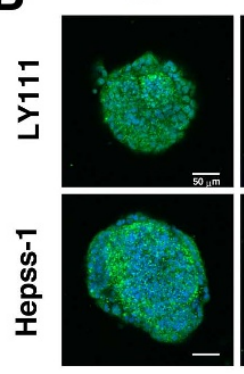

C Nanog

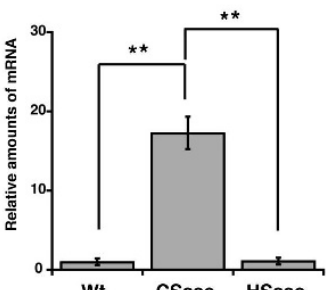

Wt CSase HSase
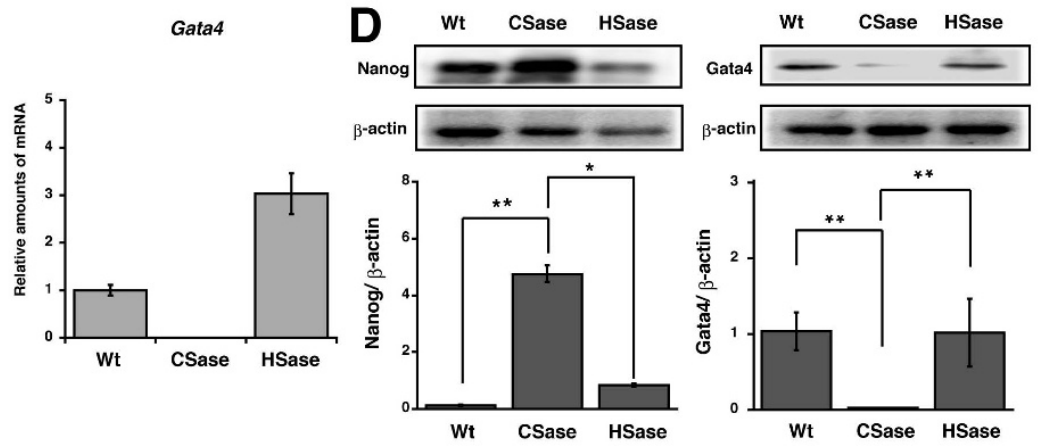

E

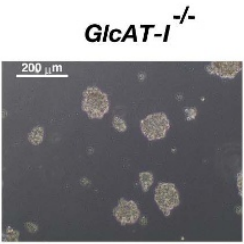

CS-A

CS-C

CS-E
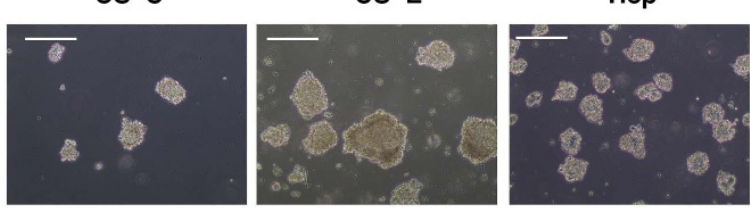

$\mathbf{F}$
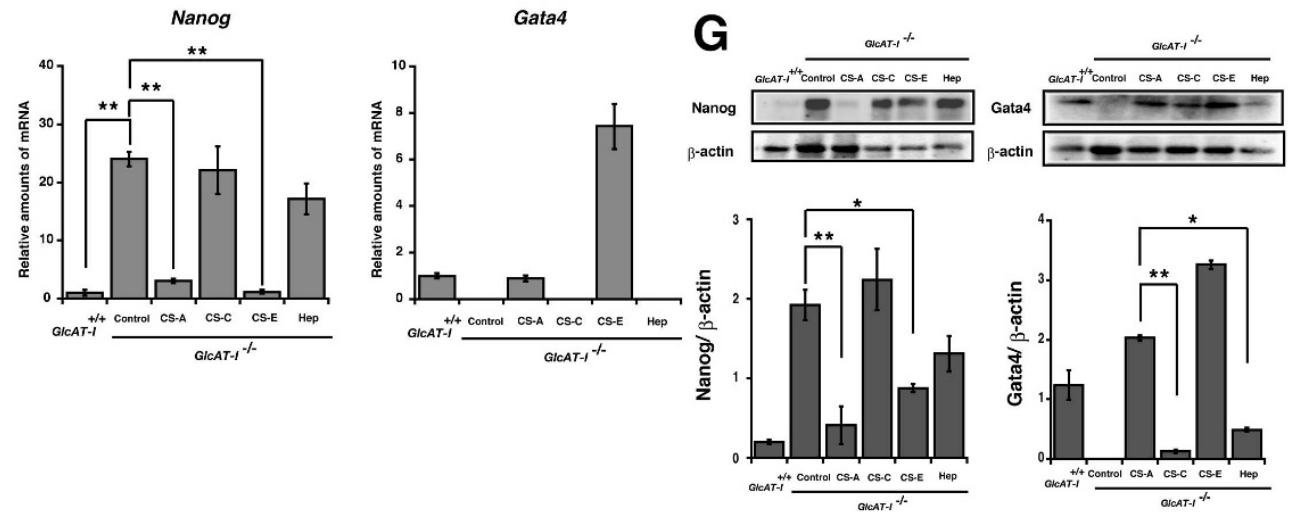

Figure $4 \mid$ Sulfation pattern of CS regulates EB differentiation. (A), Phase-contrast microscopy of day 6 Wt EBs following the addition of chondroitinase ABC (CSase) or heparitinase/heparinase (HSase) from day 0 onwards. (B), Immunofluorescence images for CS and HS chains of EB digested by continuous CSase and HSase treatment. Effective CS or HS digestion was confirmed by staining with the anti-CS antibody (LY111) (green) or anti-HS antibody (Hepss-1) (green), respectively. Nuclei were visualized with DAPI staining (blue). (C), Real-time PCR results of Nanog, the pluripotency gene, and Gata4, the differentiation marker gene, from day 6 EBs. GAPDH transcripts were used as an internal control. ${ }^{* *} \mathrm{P}<0.005$, untreated $G l c A T-I^{+/+}$ versus $G l c A T-I^{+/+}$ES cells treated with CSase, and $G l c A T-I^{+/+}$ES cells treated with CSase versus GlcAT-I $I^{+/+}$ES cells treated with HSase. (D), Western blot and quantitative analysis of the pluripotency marker, Nanog, and the differentiation marker, Gata4 from day 6 EBs. $\beta$-actin was used as a loading control. The gels have been run under the same experimental condition. The blot image was cropped around the region of interest. The results are representative of at least three independent experiments. Statistical analysis was carried out by Student's $t$ test. $* \mathrm{P}<0.01$ and $* * \mathrm{P}<0.005$. (E), Phase-contrast images of day 6 GlcAT-I ${ }^{-1-}$ EBs following addition of $10 \mu \mathrm{g} / \mathrm{ml} \mathrm{CS-A,} \mathrm{CS-C,} \mathrm{CS-E,} \mathrm{or} \mathrm{Hep} \mathrm{from} \mathrm{day} 0$ onwards. F, RT-PCR and G, western blot results of Nanog and Gata4 from EBs cultured with GAGs at day 6. The gels have been run under the same experimental condition. The blot image was cropped around the region of interest. The results are representative of at least three independent experiments. Statistical analysis was carried out by Student's $t$ test. ${ }^{*} \mathrm{P}<0.01$ and $* * \mathrm{P}<0.005$. 
A

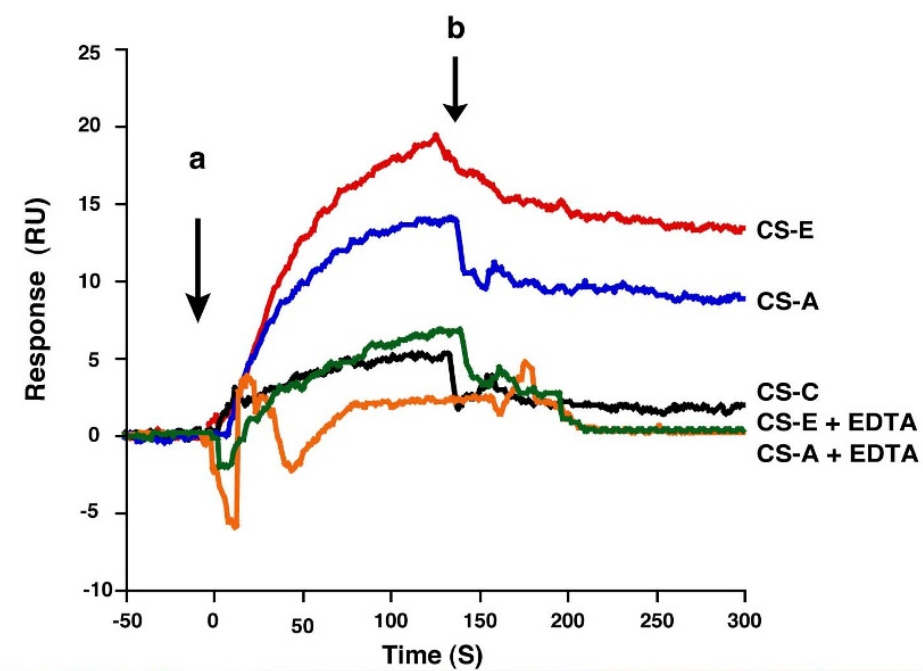

B
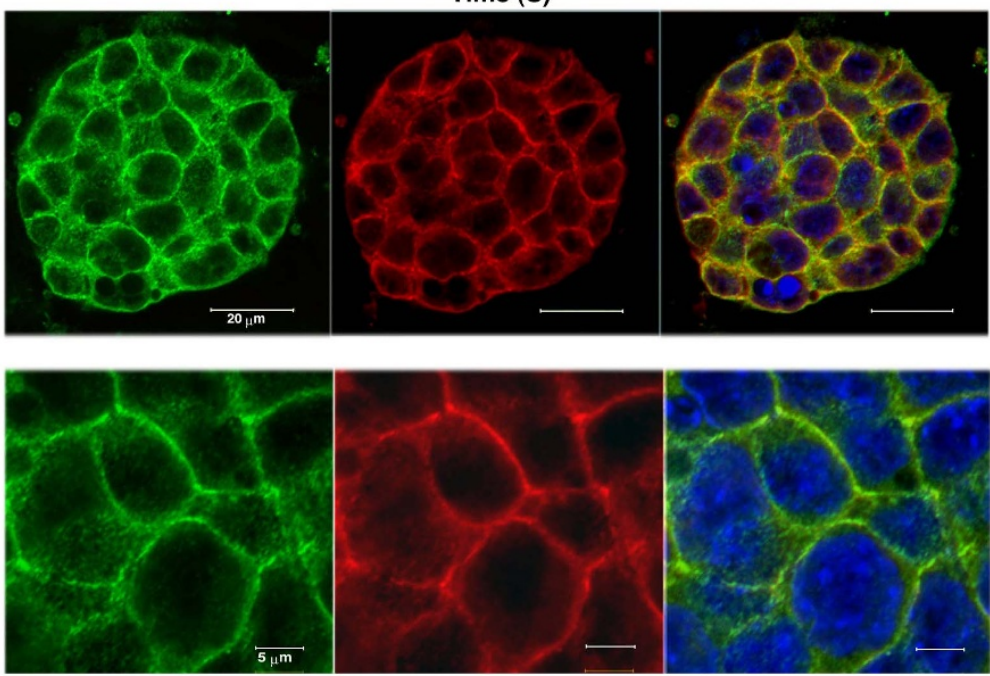

C
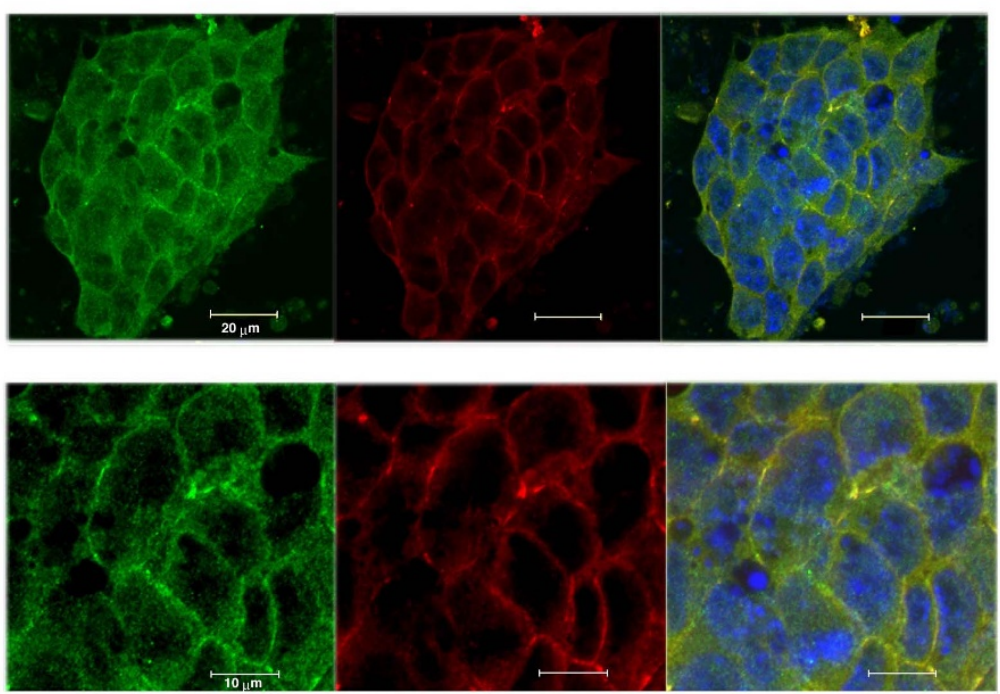

Figure 5 CS colocalizes with and binds to E-cadherin. (A), CS-A polysaccharide and CS-E polysaccharide (400 nM) were injected separately over recombinant E-cadherin- $\mathrm{Fc}$, which was immobilized on a sensor chip, in the presence of $3 \mathrm{mM} \mathrm{CaCl}_{2}$ or $3 \mathrm{mM}$ EDTA. The beginning of the association and dissociation phases is marked by the arrows "a" and "b", respectively. Wild-type ESCs were stained with (B), E-cadherin (green) and CS (LY111) (red) or (C), E-cadherin (green) and HS (Hepss-1) antibody (red). Lower panels show high-magnification images of wild-type ESCs. Nuclei were visualized with DAPI staining (blue). CS colocalized with E-cadherin. 
A

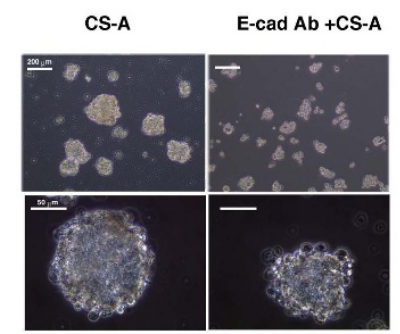

C

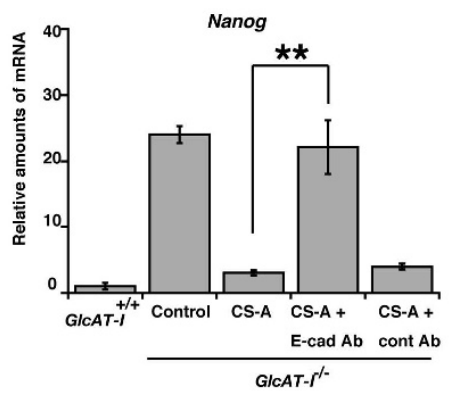

E

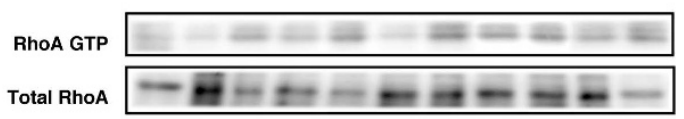

**

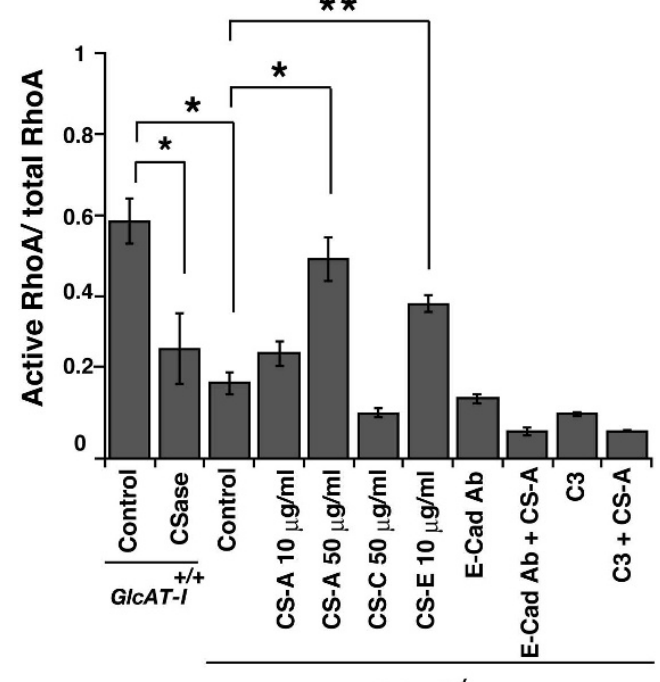

GICAT-I $^{-1}$
B

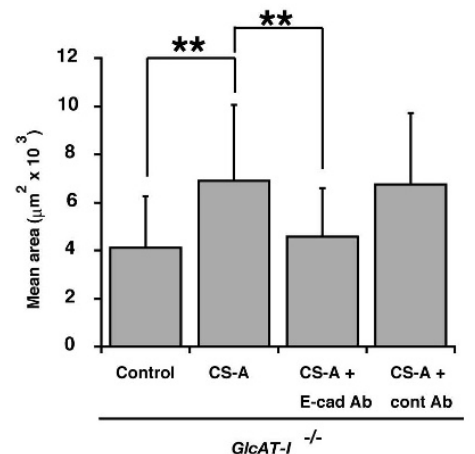

D

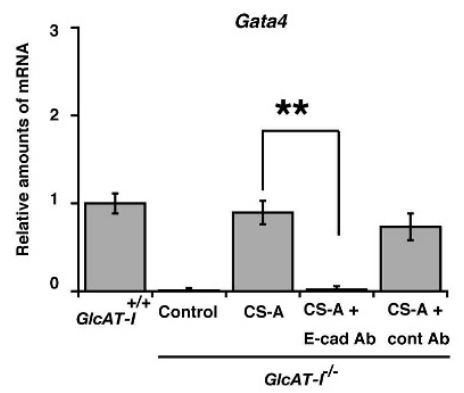

F
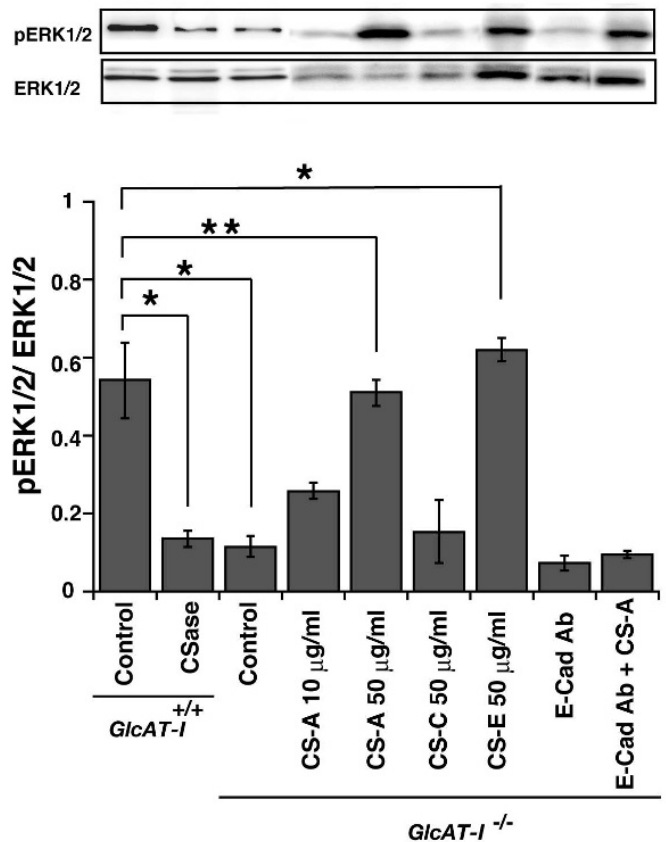

Figure $6 \mid$ CS-A and CS-E modulate E-cadherin-mediated intracellular signaling. Wild-type ESCs were treated with or without CSase and cultured. GlcAT-I ${ }^{-1-}$ ESCs were treated with CS-A polysaccharide (CS-A), CS-E polysaccharide (CS-E), CS-C polysaccharide (CS-C), anti-E-cadherin antibody (E$\mathrm{Cad} \mathrm{Ab}$ ), control IgG2b antibody (cont Ab), or RhoA inhibitor (C3). In addition, GlcAT-I-1- ESCs were treated with anti-E-cadherin antibody, control IgG2b antibody, or C3 for $1 \mathrm{~h}$, followed by addition of CS-A polysaccharide. A, Phase-contrast microscopy of day 4 EBs. Lower panels show highmagnification images of the EBs. B, Size analysis of EBs. The results revealed that the mean area of EBs, compared with that of the control group, was larger in the CS-A group and was smaller in the CS-A with E-cad antibody. Statistical analysis was carried out by Student's $t$ test. ${ }^{* *} \mathrm{P}<0.005$. C and D, Real-time PCR results for a pluripotency gene, Nanog, or a differentiation marker, GATA4, during in vitro differentiation of ESCs. GAPDH transcripts were used as an internal control. Each bar represents the mean ( \pm S.E.) values of three independent experiments. ${ }^{* *} \mathrm{P}<0.05, G l c A T-I^{-1-}$ ES cells treated with CS-A versus $\mathrm{GlcAT}_{-\mathrm{I}^{-1-}}$ treated with both CS-A and anti-E-cadherin antibody. Proteins were extracted from cells and analyzed with immunoblotting with antiRhoA antibody in E, or anti-ERK1/2 and anti-phospho-ERK1/2 antibodies in F. The gels have been run under the same experimental condition. The blot image was cropped around the region of interest. The results are representative of at least three independent experiments. Each bar represents the mean ( \pm S.E.) values of three independent experiments. E, ${ }^{*} \mathrm{P}<0.01$, Wt versus Wt ES cells treated with CSase, Wt versus GlcAT- $I^{-1-}$ ES cells, GlcAT- $I^{-1-}$ versus

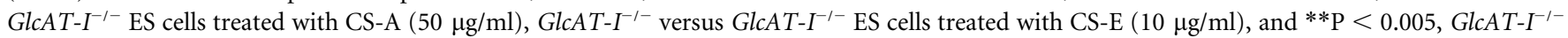

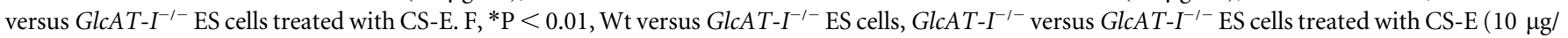

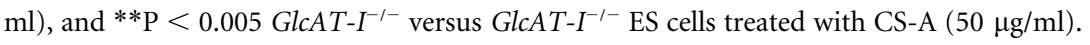


Rho-Rock signaling is attenuated in GlcAT-I ${ }^{-/-}$ESCs. In ES cells, both E-cadherin and ECM signals can regulate Rho activity ${ }^{36}$. Endogenous Rho signaling is required for the maintenance of cellcell contact in ESCs, and the level of active RhoA decreases in response to E-cadherin-mediated adhesion. In addition, upregulation of active RhoA increases ERK1/2 phosphorylation ${ }^{37}$. Therefore, intracellular signaling pathways were further analyzed to determine whether CS chains regulate ESC differentiation by binding to Ecadherin.

As shown in Fig. 6E and F, ESCs treated with CSase indeed exhibited a decrease in active RhoA and ERK1/2 phosphorylation. In GlcAT-I $I^{-1-}$ ESCs, the levels of active RhoA and ERK1/2 phosphorylation were reduced, whereas the levels of active RhoA and ERK1/2 phosphorylation were rescued when $G l c A T-I^{-1-}$ ESCs were cultured in the presence of CS-A or CS-E, but not CS-C (Fig. 6E and F). In

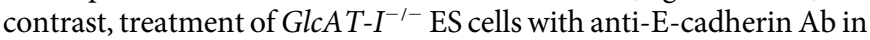
the presence of CS-A failed to increase active RhoA and ERK1/2

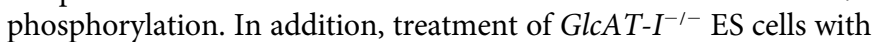
C3 transferase, which inactivates Rho proteins by ADP-ribosylation without affecting other Rho GTPases such as $\mathrm{Cdc} 42$ and $\mathrm{Rac}^{38}$, in the presence of CS-A failed to increase active RhoA. These results indicate that CS-A and CS-E bound E-cadherin with significant affinity and induced intracellular signaling downstream of E-cadherin.

\section{Discussion}

Numerous studies have shown that signaling molecules such as LIF, together with transcription factors such as Nanog, control the native pluripotency of ESCs by protecting ESCs from fibroblast growth factor (FGF)-4-mediated commitment to differentiation ${ }^{29,39-42}$. In addition, HS-mediated FGF signaling has recently been shown to be rate limiting for commitment of the PE to the neural lineage $e^{28}$. In this study, we investigated the requirement for CS in the specification and maintenance of ESCs by analyzing the consequence of loss

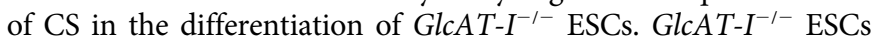
failed to differentiate in vitro and failed to maintain transcriptional and morphological characteristics of ESCs despite culture conditions promoting differentiation. Similarly, addition of CS-A or CS-E to GlcAT-I $I^{-\prime-}$ EBs rescued PE differentiation, whereas addition of CS-C, HS, or Hep did not. Thus, our results emphasized the absolute requirement of signaling mediated by CSs with specific structures for the establishment of cell-cell contact-mediated EB differentiation of ESCs, as primitive endoderm-specific markers such as Gata4 were upregulated and pluripotency markers such as Nanog were downregulated in ESCs. In addition, mesodermal and endoderm markers such as Brachyury and FoxA2 were not expressed despite addition of CS-A or CS-E to GlcAT-I I-- EBs (data not shown), because the differentiation program could not proceed in the absence of HSFGF4 signaling ${ }^{43}$. These results clearly indicate that although both CS and HS are important for ES differentiation, the functions of CS are different from those of HS in ESCs.

A recent study demonstrated that E-cadherin knockout ES cells exhibit loss of cell-cell contact and cellular proliferation and maintain an undifferentiated phenotype in the absence of $\mathrm{LIF}^{7}$, suggesting that cell-surface E-cadherin positively regulates the differentiation of ESCs. Conversely, enhanced iPS cell generation can be achieved by overexpression of E-cadherin, and knockdown of endogenous Ecadherin or abrogation of cell-cell contact by E-cadherin inhibition results in reduced reprogramming efficiency ${ }^{8}$. In addition, differentiation of ESCs is associated with the downregulation of cell surface Ecadherin $^{44}$, and in a screen for cell adhesion molecules, E-cadherin is upregulated during reprogramming ${ }^{9}$. Moreover, E-cadherin is essential for the maintenance of pluripotency of $\mathrm{mESCs}^{10}$. The reasons for these opposite functions in which ES cells exit the proliferative state and show enhanced pluripotency through E-cadherin are unclear. However, the present finding that CS is a novel determinant in controlling the functional integrity of ESCs by binding to E-cadherin can account for the opposite functions of E-cadherin as follows (Fig. 7). Lack of CS causes abnormal, ubiquitous, and constitutively high expression of Nanog; conversely, addition of CS-A or CS-E to GlcAT-I $I^{-1-}$ ESCs causes substantial reduction in the expression of Nanog and increased induction of the PE marker Gata4. In addition, the effect of addition of CS-A or CS-E to GlcAT-I ${ }^{-1-}$ ESCs was completely blocked by anti-E-cadherin $\mathrm{Ab}$. These data strongly suggest that CS is a novel determinant of the ESC state through its interaction with E-cadherin and that it controls the balance between self-renewal and differentiation. We propose that interaction between CS and E-cadherin stimulates the tendency of ESCs to differentiate by counterbalancing signaling pathways and transcription factors that promote native pluripotency. However, homophilically interacting E-cadherin or lack of E-cadherin inhibits the differentiation of ESCs due to the disruption of the binding of CS to E-cadherin (Fig. 7).

This study also showed that CS binding to E-cadherin promotes ESC differentiation through control of the Rho signaling pathway. Rho signaling is required for the maintenance of cell-cell contacts in mESCs $^{37}$. In addition, inhibition of Rho kinase efficiently improves the derivation of ESCs ${ }^{45}$. When Wt ESCs were treated with CSase, the Nanog expression increased and Rho signaling was downregulated, compared to the findings for untreated Wt ESCs (Fig. 4C and Fig. 6E). In addition, GlcAT-I $I^{-1-}$ ESCs lacking CS exhibited abnormalities similar to those exhibited by ESCs overexpressing or lacking E-cadherin. Because CS is also important for E-cadherin-mediated reprogramming of ESCs, CSase or an inhibitor of CS biosynthesis may be useful for the culture of ESCs and induction of iPS cells.

\section{Methods}

Materials. Proteus vulgaris chondroitinase ABC (CSase; EC 4.2.2.4), Flavobacterium heparinum heparitinase and heparinase, the monoclonal antibodies Hepss- 1 and LY111, CS-A from whale cartilage, CS-C from shark cartilage, CS-E from squid cartilage, and HS from bovine kidneys were purchased from Seikagaku Corp. (Tokyo, Japan). The recombinant E-cadherin Fc chimera was purchased from R\&D Systems (Minneapolis, MN). Heparin from porcine intestinal mucosa was purchased from Sigma. The anti-E-cadherin (ECCD-1) antibody was purchased from Takara. Antibodies against ERK1/2 and phospho-ERK1/2 were purchased from Cell Signaling Technology (Boston, MA).

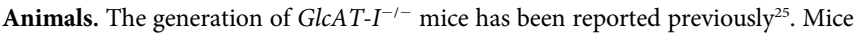
were kept under pathogen-freeconditions in an environmentally controlled, clean room at the Institute of Laboratory Animals, Kobe Pharmaceutical University; animals were maintained on standard rodent food and on a 12-h light/12-h dark cycle. All experiments were conducted according to the institutional ethical guidelines for animal experiments and safety guidelines for gene manipulation experiments. All animal procedures were approved by the Kobe Pharmaceutical University Committee on Animal Research and Ethics.

Isolation of ESC lines. GlcAT-I $I^{-1-}$ and $G l c A T-I^{+/+}$ES cells were isolated from the inner cell mass of explanted blastocysts derived from heterozygous intercrosses $\left(G l c A T-I^{+/-} \times G l c A T-I^{+/-}\right)$. Genotypes were determined with PCR using wild-type allele-specific primers (primers a: 5'-CTGAGGATATCCCAGTTGC-3' and b: $5^{\prime}$-ACATAGATAGTAGGCAGGGCC-3') or mutant allele-specific primers for the neo gene (primers c: 5'-GTTGTCACTGAAGCGGGAAGG-3' and d: 5' GAAGAACTCGTCAAGAAGGCGATAG-3'). PCR was carried out with Go-Taq Flexi DNA polymerase (Promega, Madison, WI) for 30 cycles at $96^{\circ} \mathrm{C}$ for $20 \mathrm{~s}, 56^{\circ} \mathrm{C}$ for $20 \mathrm{~s}$, and $72^{\circ} \mathrm{C}$ for $30 \mathrm{~s}$.

ESC culture. ESCs were maintained on a monolayer of mitomycin-C-inactivated mouse fibroblasts in the presence of knockout DMEM supplemented with $20 \%$ KnockOut ${ }^{\mathrm{TM}}$ Serum Replacement, 100 mM 2-mercaptoethanol, MEM non-essential amino acids, $2 \mathrm{mM}$ L-glutamine, $1 \mathrm{mM}$ pyruvate, and $100 \mathrm{U} / \mathrm{ml}$ LIF ESGRO (Millipore). The medium was changed every day, and ES cells were passaged every second day.

Analyses of the disaccharide composition of GAGs. GAGs from feeder-free ES cells were prepared as described previously ${ }^{17}$. The purified GAG fraction was digested with CSase or a mixture of heparinase and heparitinase, and the digests were then derivatized with 2-aminobenzamide and analyzed with high-performance liquid chromatography as described ${ }^{17}$.

In vitro differentiation of ES cells. Before beginning differentiation experiments, the ES cell cultures were depleted of feeder cells by incubating trypsinized cells in ES cell medium on culture dishes for $30 \mathrm{~min}$, during which only the feeder cells attached to 


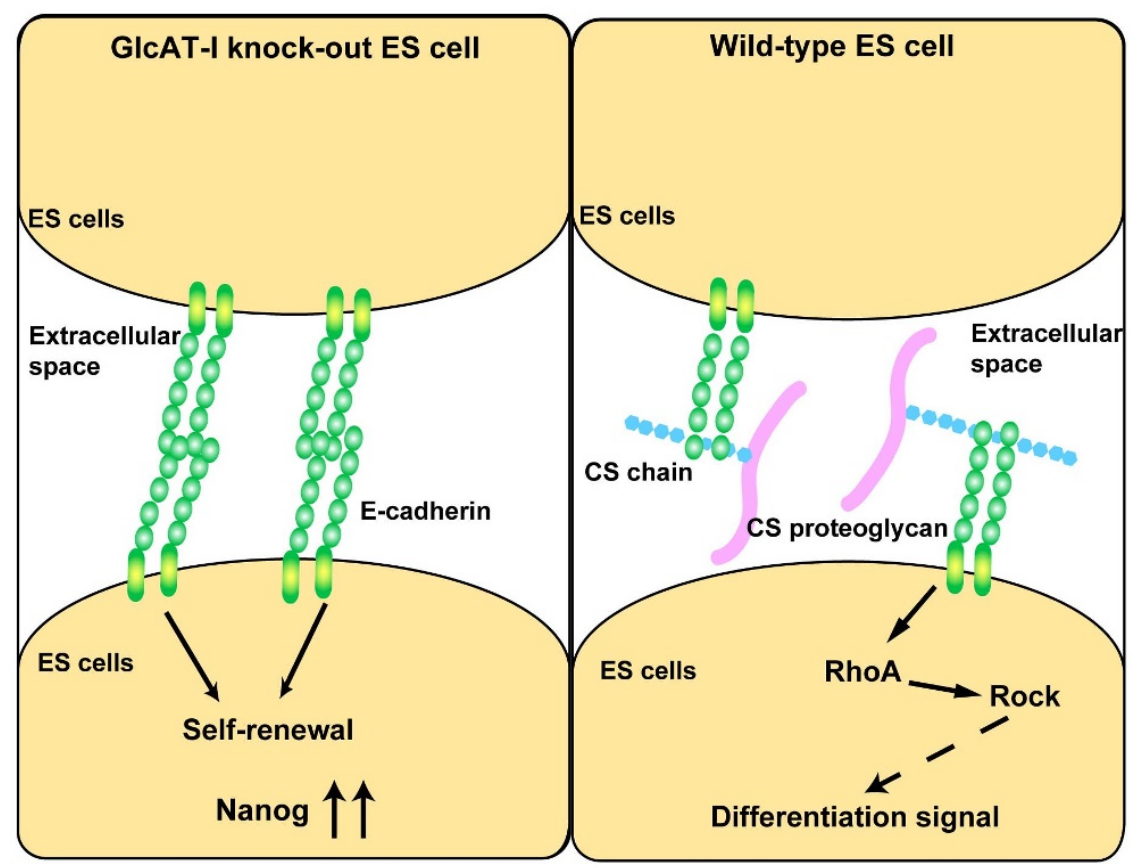

Figure $7 \mid$ CS controls the functional integrity of ESCs by binding to E-cadherin. In GlcAT-I $I^{-1-}$ ESCs, homophilically interacting E-cadherin inhibits the differentiation of ESCs due to the disruption of the binding of CS to E-cadherin. On the other hand, in Wt ESCs, the interaction between CS and Ecadherin stimulates the tendency of ESCs to differentiate by counterbalancing signaling pathways and transcription factors that promote native pluripotency.

the dish. Subsequently, the remnant ES cells were plated on gelatin-coated dishes, cultured overnight, and feeder-depleted again before they were counted and plated according to the various differentiation protocols. Differentiation in adherent monolayer culture was achieved in $20 \%$ KnockOut $^{\mathrm{TM}}$ Serum Replacement without LIF. For differentiation into EBs, ESCs were transferred into bacterial dishes at 5000 cells/ml in $20 \%$ KnockOut $^{\mathrm{TM}}$ Serum Replacement without LIF. Differentiating EBs and ESCs were exposed to CS-A, CS-C, CS-E, Hep, or HS. For size analysis, mean diameters were measured using morphological analysis software (Mac SCOPE; Mitani Corp., Tokyo, Japan) and calculated from more than five sample EBs per field in 10 fields. At least $300 \mathrm{EBs}$ were analyzed for each sample.

Self-renewal assay. ES cells were seeded at clonal density and cultured for $5 \mathrm{~d}$ in ES medium with or without LIF. The culture medium was removed, and the cells were washed with phosphate-buffered saline (PBS) and tested for alkaline phosphatase activity using an alkaline phosphatase kit (Millipore). One hundred colonies were scored, and the percentage of AP-positive colonies was calculated. Dome-shaped colonies with tightly packed AP-positive cells were considered undifferentiated. Colonies with a mixture of stained and unstained colonies and with flattened and non-uniform morphology were considered differentiated ${ }^{31}$.

Immunofluorescence. For immunofluorescence, cells were fixed in $4 \%$ paraformaldehyde for $10 \mathrm{~min}$ and blocked in 3\% BSA for $60 \mathrm{~min}$, with PBS washes between all steps. For whole-mount EB immunofluorescence, EBs after treatment with CSase or HSase were washed in PBS, fixed for $1 \mathrm{~h}$ in $4 \% \mathrm{PFA}$ at $4^{\circ} \mathrm{C}$ and washed three times with wash buffer $(150 \mathrm{mM} \mathrm{NaCl}, 1 \mathrm{mg} / \mathrm{ml} \mathrm{BSA}, 0.1 \%$ Tween- $20,50 \mathrm{mM}$ Tris-HCl pH 7.5). EBs were permeabilized for $30 \mathrm{~min}$ in $2 \%$ TritonX-100, refixed in $4 \%$ PFA for $20 \mathrm{~min}$, and then blocked in wash buffer for $2 \mathrm{~h}^{46}$. Antibodies were diluted in blocking solution. The primary antibodies and the corresponding dilutions used in this study were Hepss- 1 ( $1: 1000$; Seikagaku Corp.), LY111 (1:500; Seikagaku Corp.), Oct3/4 (sc-5279, 1:200; Santa Cruz Biotechnology, Inc.), Nanog (1:500; ReproCell), SOX2 (\#245610, $1: 150$, R\&D Systems), and E-cadherin (ECDD-2, $1: 100$; Takara). Appropriate secondary antibodies (Alexa488-conjugated $1: 200$, Alexa568-conjugated $1: 200$ ) were obtained from Invitrogen. To visualize nuclei, cells were incubated in DAPI (Dojindo) for $10 \mathrm{~min}$ at room temperature. Fluorescent images were obtained using a laser-scanning confocal microscope LSM710 (Carl Zeiss).

Rho activation assay. Rho activity was determined by measuring RhoA-GTP binding to the glutathione $S$-transferase-RhoA-binding domain in a pull-down assay using a Rho assay reagent (Upstate) according to the manufacturer's instructions.

Protein extraction and western blot analysis. Cells were lysed with immunoprecipitation buffer containing $0.5 \mathrm{mM}$ phenylmethylsulfonyl fluoride, complete protease inhibitor mixture (Roche), $1 \%$ Triton X-100, and $1 \mathrm{mM}$ sodium orthovanadate. Cell lysates were centrifuged at $12,000 \times g$ for $20 \mathrm{~min}$ at $4{ }^{\circ} \mathrm{C}$, and the supernatants were stored at $-80^{\circ} \mathrm{C}$. Protein quantification was performed using the bicinchoninic acid protein assay reagent (Pierce). Protein aliquots $(3 \mu \mathrm{g})$ were denatured in SDS sample buffer and electrophoresed on $10 \%$ polyacrylamide-SDS gels. The membranes were immunoblotted using a primary antibody against SOX2 (\#245610, $1: 1000$, R\&D Systems), Gata4 ( $1: 200 ; \mathrm{H}-112$, Santa Cruz Biotechnology, Inc.), Oct3/4 (sc-5279, $1: 200$; Santa Cruz Biotechnology, Inc.), Nanog ( $1: 1000$; ReproCell), RhoA (1:200; Santa Cruz Biotechnology, Inc.), ERK1/2 (\#9102, 1:200; Cell Signaling), phospho-ERK1/2 (\#9101, 1:200; Cell Signaling), and $\beta$-actin $(1: 1000 ;$ Sigma). The antigen-antibody complexes were visualized using appropriate secondary antibodies (Sigma) and the enhanced chemiluminescence detection system, as recommended by the manufacturer (GE Healthcare).

ES cell culture with CSase or HSase. Before beginning differentiation experiments, the ES cell cultures were depleted of feeder cells by incubating trypsinized cells in ES cell medium on culture dishes for $30 \mathrm{~min}$, during which only the feeder cells attached to the dish. Subsequently, the remnant ES cells were plated on gelatin-coated dishes, cultured overnight, and feeder-depleted again in ES cell medium with the addition of CSase (10 mIU) or HSase (Heparinase and heparitinase, $5 \mathrm{mIU}$ each) on culture dishes for $60 \mathrm{~min}$ before they were counted. ESCs were transferred into bacterial dishes at 5000 cells $/ \mathrm{ml}$ in $20 \%$ KnockOut ${ }^{\mathrm{TM}}$ Serum Replacement without LIF and with the addition of CSase (10 mIU) or HSase (Heparinase and heparitinase, $5 \mathrm{mIU}$ each). The medium with CSase or HSase was changed every second day.

Quantitative real-time RT-PCR. Total RNA was extracted from ES cells using TRIzolR reagent (Invitrogen). cDNA was synthesized from $1 \mu \mathrm{g}$ of total RNA by using Moloney murine leukemia virus reverse transcriptase (Promega) and a random nonamer primer (TaKaRa Bio Inc., Shiga, Japan). The primer sequences were: Nanog, forward primer $5^{\prime}$-AGAACAAGGTCCTTGCCA-3' and reverse primer 5'-TGCTTATAGCTCAGGTTCAGAATG-3'; Oct4, forward primer 5'-CATGTTTCTGAAGTGCCCG-3' and reverse primer $5^{\prime}$-GTAGCCTCATACTCTTCTCGT-3'; Sox2, forward primer 5'-GCCGAGTGGAAACTTTTGTCC-3' and reverse primer $5^{\prime}$-CGGGAAGCGTGTACTTATCCTT-3'; Nestin, forward primer $5^{\prime}$-CCTGAACACACACTAGAGACA- $3^{\prime}$ and reverse primer $5^{\prime}$ - TTCAAGCATCTGGTCCTCG-3'; Gata4, forward primer 5' -GCCAACTGTGGCATTACTTTAT$3^{\prime}$ and reverse primer $5^{\prime}$-GACAATGTTAACGGGTTGTGGA-3'; Foxc1, forward primer $5^{\prime}$-CCCCGGACAAGAAGATCACTC-3' and reverse primer $5^{\prime}$-AGGTTGTGCCGTATGCTGTTC-3'; Brachyury, forward primer 5' -TCCACACACGGCTGTGA-3' and reverse primer 5' - CCGAGGCTAGACCAGTTA- ${ }^{\prime}$; and glyceraldehyde-3-phosphate dehydrogenase (GAPDH), forward primer 5' -CATCTGAGGGCCCACTG- ${ }^{\prime}$ and reverse primer 5'-GAGGCCATGTAGGCCATGA- ${ }^{\prime}$. Quantitative real-time RT-PCR was performed using FastStart DNA Master plus SYBR Green I in a LightCycler ST300 (Roche). The expression level of each gene was normalized to that of the GAPDH transcript. 
Interaction analysis. The binding of CS-A, CS-C, or CS-E polysaccharide to Ecadherin was examined using the BIAcore J system (GE Healthcare) as described previously ${ }^{47}$, with slight modifications. Briefly, recombinant E-cadherin-Fc was immobilized on a CM5 sensor chip (GE Healthcare), according to the manufacturer's instructions. A series of CS-A or CS-E concentrations ranging from 20 to $400 \mathrm{nM}$ in running buffer was applied to the flow cells, and changes in resonance units were recorded. Data were analyzed using the BIAevaluation 3.0 software (GE Healthcare) using a 1:1 Langmuir binding model.

Statistical analysis. Student's $t$-test was used for determination of statistical significance throughout the study. Differences were considered to be significant with a $\mathrm{P}$ value less than 0.01 .

1. Evans, M. J. \& Kaufman, M. H. Establishment in culture of pluripotential cells from mouse embryos. Nature 292, 154-156 (1981).

2. Martin, G. R. Isolation of a pluripotent cell line from early mouse embryos cultured in medium conditioned by teratocarcinoma stem cells. Proc. Natl. Acad. Sci. U.S.A. 78, 7634-7638 (1981).

3. Keller, G. Embryonic stem cell differentiation. Emergence of a new era in biology and medicine. Genes Dev. 19, 1129-1155 (2005).

4. Bissell, M. J., Kenny, P. A. \& Radisky, D. C. Microenvironmental regulators of tissue structure and function also regulate tumor induction and progression: the role of extracellular matrix and its degrading enzymes. Cold Spring Harb. Symp. Quant. Biol. 70, 343-356 (2005).

5. Daley, W. P., Peters, S. B. \& Larsen, M. Extracellular matrix dynamics in development and regenerative medicine. J. Cell Sci. 121, 255-264 (2008).

6. Chou, Y. F. et al. The growth factor environment defines distinct pluripotent ground states in novel blastocyst-derived stem cells. Cell 135, 449-461 (2008)

7. Soncin, F. et al. Abrogation of E-cadherin-mediated cell-cell contact in mouse embryonic stem cells results in reversible LIF-independent self-renewal. Stem Cells 27, 2069-2080 (2009).

8. Chen, T. et al. E-cadherin-mediated cell-cell contact is critical for induced pluripotent stem cell generation. Stem Cells 28, 1315-1325 (2010).

9. Li, R. et al. A mesenchymal-to-epithelial transition initiates and is required for the nuclear reprogramming of mouse fibroblasts. Cell Stem Cell 7, 51-63 (2010).

10. Redmer, T. et al. E-cadherin is crucial for embryonic stem cell pluripotency and can replace OCT4 during somatic cell reprogramming. EMBO Reports 12, 720-726 (2011).

11. Kitagawa, H. et al. Molecular Cloning and Expression of Glucuronyltransferase I Involved in the Biosynthesis of the Glycosaminoglycan-Protein Linkage Region of Proteoglycans. J. Biol. Chem. 273, 6615-16618 (1998).

12. Tone, Y., Kitagawa, H., Oka, S., Kawasaki, T. \& Sugahara, K. Characterization of recombinant human glucuronyltransferase I involved in the biosynthesis of the glycosaminoglycan-protein linkage region of proteoglycans. FEBS Lett. 459, 415-420 (1999).

13. McCormick, C. et al. The putative tumour suppressor EXT1 alters the expression of cell-surface heparan sulfate. Nat. Genet. 19, 158-161 (1998).

14. Lind, T., Tufaro, F., McCormick, C., Lindahl, U. \& Lidholt, K. The putative tumor suppressors EXT1 and EXT2 are glycosyltransferases required for the biosynthesis of heparan sulfate. J. Biol. Chem. 273, 26265-26268 (1998).

15. Kitagawa, H., Uyama, T. \& Sugahara, K. Molecular Cloning and Expression of a Human Chondroitin Synthase. J. Biol. Chem. 276, 38721-38726 (2001).

16. Izumikawa, T., Uyama, T., Okuura, Y., Sugahara, K. \& Kitagawa, H. Involvement of chondroitin sulfate synthase-3 (chondroitin synthase-2) in chondroitin polymerization through its interaction with chondroitin synthase-1 or chondroitin-polymerizing factor. Biochem. J. 403, 545-552 (2007).

17. Izumikawa, T. et al. Identification of Chondroitin Sulfate Glucuronyltransferase as Chondroitin Synthase-3 Involved in Chondroitin Polymerization: CHONDROITIN POLYMERIZATION IS ACHIEVED BY MULTIPLE ENZYME COMPLEXES CONSISTING OF CHONDROITIN SYNTHASE FAMILY MEMBERS. J. Biol. Chem. 283, 11396-11406 (2008).

18. Kitagawa, H., Izumikawa, T., Uyama, T. \& Sugahara, K. Molecular Cloning of a Chondroitin Polymerizing Factor That Cooperates with Chondroitin Synthase for Chondroitin Polymerization. J. Biol. Chem. 278, 23666-23671 (2003).

19. Sugahara, K. \& Kitagawa, H. Recent advances in the study of the biosynthesis and functions of sulfated glycosaminoglycans. Curr. Opin. Struct. Biol. 10, 518-527 (2000).

20. Kitagawa, H., Tsutsumi, K., Tone, Y. \& Sugahara, K. Developmental regulation of the sulfation profile of chondroitin sulfate chains in the chicken embryo brain. J. Biol. Chem. 272, 31377-31381 (1997)

21. Thiele, H. et al. Loss of chondroitin 6-O-sulfotransferase-1 function results in severe human chondrodysplasia with progressive spinal involvement. Proc. Natl. Acad. Sci. U. S. A. 101, 10155-10160 (2004).

22. Klüppel, M., Wight, T. N., Chan, C., Hinek, A. \& Wrana, J. L. Maintenance of chondroitin sulfation balance by chondroitin-4-sulfotransferase 1 is required for chondrocyte development and growth factor signaling during cartilage morphogenesis. Development 132, 3989-4003 (2005).

23. Miyata, S., Komatsu, Y., Yoshimura, Y., Taya, C. \& Kitagawa, H. Persistent cortical plasticity by upregulation of chondroitin 6-sulfation. Nat. Neurosci. 15, 414-422 (2012).
24. Esko, J. D. \& Lindahl, U. Molecular diversity of heparan sulfate. J. Clin. Invest. 108, 169-173 (2001).

25. Izumikawa, T. et al. Impairment of embryonic cell division and glycosaminoglycan biosynthesis in glucuronyltransferase-I-deficient mice. J. Biol. Chem. 285, 12190-1219 (2010).

26. Lin, X. et al. Disruption of gastrulation and heparan sulfate biosynthesis in EXT1deficient mice. Dev. Biol. 224, 299-311 (2000).

27. Stickens, D., Zak, B. M., Rougier, N., Esko, J. D. \& Werb, Z. Mice deficient in Ext2 lack heparan sulfate and develop exostoses. Development 132, 5055-5068 (2005).

28. Forsberg, M. et al. Undersulfation of heparan sulfate restricts differentiation potential of mouse embryonic stem cells. J. Biol. Chem. 287, 10853-10862 (2012)

29. Lanner, F. \& Rossant, J. The role of FGF/Erk signaling in pluripotent cells. Development 137, 3351-3360 (2010).

30. Pickford, C. E. et al. Specific glycosaminoglycans modulate neural specification of mouse embryonic stem cells. Stem Cells 29, 629-640 (2011).

31. Kraushaar, D. C., Yamaguchi, Y. \& Wang, L. Heparan sulfate is required for embryonic stem cells to exit from self-renewal. J Biol. Chem. 285, 5907-5916 (2010).

32. Uyama, T., Kitagawa, H., Tamura, J. \& Sugahara, K. Molecular Cloning and Expression of Human Chondroitin $N$-Acetylgalactosaminyltransferase: THE KEY ENZYME FOR CHAIN INITIATION AND ELONGATION OF CHONDROITIN/DERMATAN SULFATE ON THE PROTEIN LINKAGE REGION TETRASACCHARIDE SHARED BY HEPARIN/HEPARAN SULFATE. J. Biol. Chem. 277, 8841-8846 (2002).

33. Uyama, T. et al. Molecular Cloning and Expression of a Second Chondroitin NAcetylgalactosaminyltransferase Involved in the Initiation and Elongation of Chondroitin/Dermatan Sulfate. J. Biol. Chem. 278, 3072-3078 (2003).

34. Mountford, P., Nichols, J., Zevnik, B., O’Brien, C. \& Smith, A. Maintenance of pluripotential embryonic stem cells by stem cell selection. Reprod. Fertil. Dev. 10, 527-533 (1998).

35. Koike, T., Izumikawa, T., Tamura, J. \& Kitagawa, H. Chondroitin sulfate-E finetunes osteoblast differentiation via ERK1/2, Smad 3 and Smad1/5/8 signaling by binding to N-cadherin and cadherin-11. Biochem. Biophys. Res. Commun. 420, 523-529 (2012)

36. Xu, Y. et al. Revealing a core signaling regulatory mechanism for pluripotent stem cell survival and self-renewal by small molecules. Proc. Natl. Acad. Sci. U. S. A. 107, 8129-8134 (2010).

37. Harb, N., Archer, T. K. \& Sato, N. The Rho-Rock-Myosin signaling axis determines cell-cell integrity of self-renewing pluripotent stem cells. PLoS One. 3, e3001 (2008).

38. Fukata, M. \& Kaibuchi, K. Rho-family GTPases in cadherin-mediated cell-cell adhesion. Nat. Rev. Mol. Cell Biol. 2, 887-897 (2001).

39. Chambers, I. et al. Nanog safeguards pluripotency and mediates germline development. Nature 450, 1230-1234 (2007).

40. Niwa, H., Ogawa, K., Shimosato, D. \& Adachi, K. A parallel circuit of LIF signalling pathways maintains pluripotency of mouse ES cells. Nature 460 118-122 (2009).

41. Kunath, T. et al. FGF stimulation of the Erk1/2 signalling cascade triggers transition of pluripotent embryonic stem cells self-renewal to lineage commitment. Development 134, 2895-2902 (2007).

42. Nichols, J., Silva, J., Roode, M. \& from Smith, A. Suppresssion of Erk signaling promotes ground state pluripotency in the mouse embryos. Cell Stem Cell $\mathbf{8}$, 318-325 (2009)

43. Kang, M., Piliszek, A., Artus, J. \& Hadjantonakis, A. K. FGF4 is required for lineage restriction and salt-and-pepper distribution of primitive endoderm factors but not their initial expression in the mouse. Development 140, 267-279 (2013).

44. Spencer, H. L. et al. E-cadherin inhibits cell surface localization of the promigratory 5T4 oncofetal antigen in mouse embryonic stem cells. Mol. Biol. Cell 18, 2838-2851 (2007)

45. Zhang, P., Wu, X., Hu, C., Wang, P. \& Li, X. Rho kinase inhibitor Y-27632 and Accutase dramatically increase mouse embryonic stem cell derivation. In Vitro Cell Dev. Biol. Anim. 8, 30-36 (2012).

46. Richard, L. C. et al. Homogeneous and organized differentiation within embryoid bodies induced by microsphere-mediated delivery of small molecules. Biomaterials 13, 2507-2515 (2009).

47. Mikami, T., Yasunaga, D. \& Kitagawa, H. Contactin-1 is a functional receptor for neuroregulatory chondroitin sulfate-E. J. Biol. Chem. 284, 4494-4499 (2009).

\section{Acknowledgments}

This work was supported in part by the Ministry of Education, Culture, Sports, Science, and Technology (MEXT)-Supported Program for the Strategic Research Foundation at Private Universities, 2012-2017 (to H.K.) and a Grant-in-Aid for Scientific Research in Innovative Areas (No. 23110003 to H.K., Deciphering sugar chain-based signals regulating integrative neuronal functions) and for Scientific Research B (No. 25293014 to H.K.) from the Ministry of Education, Culture, Sports, Science, and Technology (MEXT) of Japan. We thank Masahide Asano for advice about isolation of the ES cells and Megumi Okada for technical assistance. 


\section{Author contributions}

T.I. and B.S. performed the research. T.I. and H.K. analyzed the data, designed the research, and wrote the manuscript. H.K. conceived the ideas.

\section{Additional information}

Supplementary information accompanies this paper at http://www.nature.com/ scientificreports

Competing financial interests: The authors declare no competing financial interests.
How to cite this article: Izumikawa, T., Sato, B. \& Kitagawa, H. Chondroitin Sulfate Is Indispensable for Pluripotency and Differentiation of Mouse Embryonic Stem Cells. Sci. Rep. 4, 3701; DOI:10.1038/srep03701 (2014)

(c) (i) (2) This work is licensed under a Creative Commons AttributionNonCommercial-ShareAlike 3.0 Unported license. To view a copy of this license, visit http://creativecommons.org/licenses/by-nc-sa/3.0 